\title{
The effect of zero-ischaemia laparoscopic minimally invasive partial nephrectomy using the modified sequential preplaced suture renorrhaphy technique on long-term renal functions
}

\author{
Mehmet Giray Sönmez ${ }^{1}$, Cengiz Kara² \\ ${ }^{1}$ Department of Urology, Meram Medical Faculty, Necmettin Erbakan University, Konya, Turkey \\ ${ }^{2}$ Department of Urology, Medical Park Ankara Hospital, Ankara, Turkey
}

Videosurgery Miniinv 2017; 12 (3): 257-263

DOI: https://doi.org/10.5114/wiitm.2017.67136

\begin{abstract}
Introduction: Laparoscopic minimally invasive partial nephrectomy (MIPN) is the preferred technique in renal surgery, especially T1 phase kidney tumours, and it is recommended for the protection of renal functions in methods that do not involve ischaemia.

Aim: To evaluate long-term renal functions of zero-ischaemia laparoscopic MIPN patients who underwent a modified sequential preplaced suture renorrhaphy technique.

Material and methods: In a total of 17 renal units in 16 patients with kidney tumours that were determined incidentally and did not cause any complaints, the masses were extracted via laparoscopic partial nephrectomy (LPN) using the modified sequential preplaced suture renorrhaphy technique. Creatinine and estimated glomerular filtration rate (eGFR) values of the patients were measured preoperatively and on the first day and after 12 months postoperatively, and the results were compared.

Results: The differences between the pre- and postoperative values were statistically significant $(p=0.033, p=$ $0.045)$, but the changes in postoperative creatinine and eGFR values were clinically insignificant. While the differences between preoperative and first-day postoperative creatinine and eGFR values were found to be statistically significant ( $p=0.039, p=0.042$, respectively), a statistically significant difference was not detected between preoperative and 12-month postoperative creatinine and eGFR values $(p=0.09, p=0.065$, respectively). The global percentage of functional recovery was measured as $92.5 \%$ on the first day and $95.9 \%$ at the $12^{\text {th }}$ month.

Conclusions: The modified sequential preplaced suture renorrhaphy technique is an effective, reliable method for avoiding complications and preserving renal functions and nephrons in appropriate patients.
\end{abstract}

Key words: laparoscopy, minimally invasive partial nephrectomy, renal functions, sequential preplaced suture renorrhaphy

\section{Introduction}

Through radiological examinations, such as ultrasonography, computed tomography (CT) and magnetic resonance imaging (MRI), which are commonly used in Turkey, kidney tumours can be detected at a rather early phase $[1,2]$. Thus, most of the new- ly defined kidney tumours are incidental masses determined due to non-urological complaints, and a significant number of these are small $(<4 \mathrm{~cm})$, early-phase and low-metastasis-potential tumours [3]. Partial nephrectomy (PN) is the reference standard for the management of clinical T1a renal masses, 
and its indications have recently been expanded to include $\mathrm{T} 1 \mathrm{~b} / \mathrm{T} 2$ renal tumours, even in the presence of a normal contralateral kidney [4].

Since these tumours are masses appropriate for nephron-protective surgery, open and laparoscopic PN (LPN) is applied for the treatment of such lesions. The LPN was applied transperitoneally by Winfield et al. in 1993 and in combination with the retroperitoneoscopic method by Gill et al.; since then, it has become the method that is initially preferred in many centres today $[5,6]$. The European Association of Urology Guideline's first recommendation in T1a kidney tumours is LPN [1].

The LPN is generally carried out with warm ischaemia, but since the ischaemia time can lengthen during the operation, permanent nephron damage may be caused in the kidney. However, avoiding ischaemia may cause an increase in bleeding tendency. Again, discussions continue concerning tumours for which laparoscopy should be carried out, the possible outcomes of ischaemia and whether it should or should not be applied. Minimally invasive PN (MIPN) is preferred, especially in T1-phase kidney tumours, and it is recommended for the protection of renal functions in methods without ischaemia.

In the literature, it has been observed that different results are presented when renal deficiency developing after PN is considered. Studies have shown a significant decrease in patients' glomerular filtration rate (GFR) after PN [7]. Although serum creatinine $(\mathrm{s} C r)$ level measurement is a significant marker for kidney functions, it is not a sufficient marker to show renal deficiency. The National Kidney Foundation stated that GFR is a more sensitive marker for evaluating renal functions and deficiency [8].

\section{Aim}

Since few reports on this topic exist in the literature, our aim in this study was to evaluate the long-term renal functions of zero-ischaemia laparoscopic MIPN patients who underwent the modified sequential preplaced suture renorrhaphy technique.

\section{Material and methods}

In a total of 17 renal units in 16 patients with kidney tumours that were determined incidentally and did not cause any complaints, the masses were extracted via LPN using the modified sequential preplaced suture renorrhaphy technique. Radiolog- ical imaging was carried out before surgery in all patients. Contrast $C T$ and/or MRI techniques were used to demonstrate the size of the kidney tumour, its localisation and the relationship with the veins and collecting system. The dimension of these masses, whether they were exophytic/endophytic, their proximity to the renal sinus and collecting tubules, location according to the polar lines and whether they were anterior/posterior were evaluated using the R.E.N.A.L. nephrometry system [9]. During selection, exophytic tumours with a tumour dimension of $\leq 4 \mathrm{~cm}$ and that had no relationship with the collecting system were preferred. The transperitoneal approach was applied to all patients. Postoperative complications were evaluated using the Clavien-Dindo classification system. Creatinine and estimated GFR (eGFR) values of the patients measured on the first day and $12^{\text {th }}$ month postoperatively were compared to creatinine and eGFR values measured preoperatively.

\section{Surgical technique}

Under general anaesthesia, a pneumoperitoneum was formed by entering with a laparoscopic Veress needle from $2 \mathrm{~cm}$ proximal to the anterior superior iliac crest in the front axillary line. A 100$\mathrm{mm}$ trocar was located from here, and subcostal and periumbilical $5-\mathrm{mm}$ and $10-\mathrm{mm}$ trocars were located under a $30^{\circ}$ camera view. With a transperitoneal approach, the colon was mobilized medially by incising the line of told. Located between the kidney and ureter the kidney was released, but an intervention was not made in the hilar veins. By releasing the kidney in Gerota's fascia, attention was paid to the preservation of perirenal fat tissue on the tumour. After the tumour was detected, two to four 1/0, 3/8 circle, 63-mm polyglycolic acid co-lactic acid (PGLA) liver sutures were placed before resection for haemostasis, leaving a $0.5-1 \mathrm{~cm}$ distance in the tumour base. The depth of the parenchymal area where the suture was to be made was planned approximately by measuring the tumour size in the CT and/or MRI images taken in the preoperative period in three dimensions. Leaving a security margin of $2 \mathrm{~mm}$ around the tumour, the tumour and the fat tissue above it were extracted together with the safety area, marking the area to be excised with a hook. Since we routinely leave a security margin, a frozen cross-section was not sent, to avoid increasing the bleeding duration. The tissue was taken out with a laparo- 
scopic pouch. After cauterising the tumour location, haemostasis was provided by connecting the liver sutures located previously. We observed that these haemostasis sutures significantly decreased the amount of bleeding. The surgical technique is shown in Photo 1 in detail.

\section{Statistical analysis}

Statistical comparison of creatinine and eGFR values preoperatively and on the first day and after
12 months postoperatively was carried out using Friedman's two-way analysis of variance. A $p$-value $<0.05$ was accepted as statistically significant.

\section{Results}

Modified sequential preplaced suture renorrhaphy was successfully applied in 17 renal units in 16 patients. The mean age of the patients was 61.7 (49-72) years. Out of 16 patients, 11 (68.75\%) were male and 5 (31.25\%) were female. Bilateral kidney
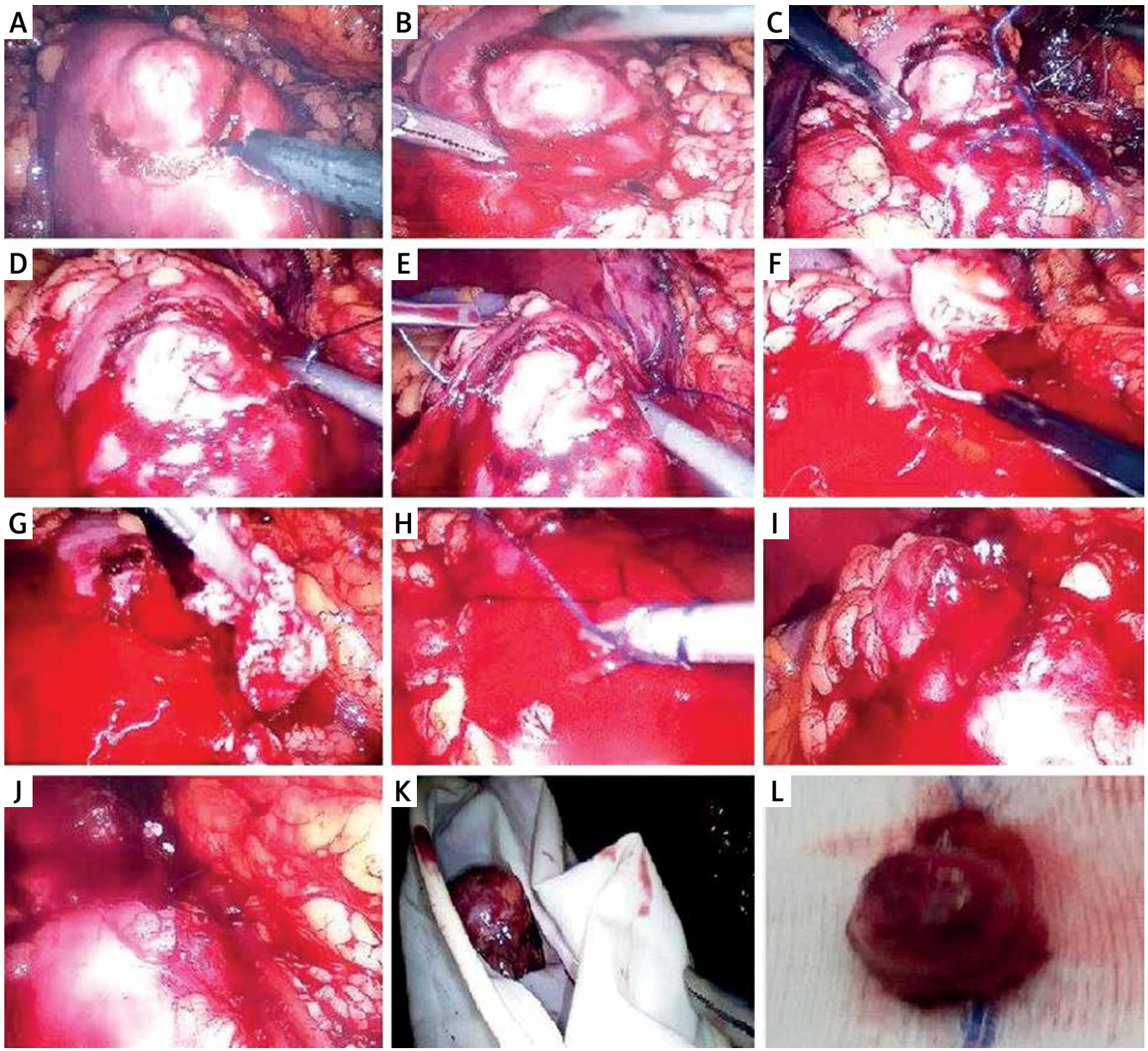

Photo 1. Surgical technique: A, B - finding the renal tumour and marking the area to be resected with cautery, C-E - making renal parenchymal sutures under the tumour with 3/8 circle, 63-mm polyglycolic acid co-lactic acid (PGLA) liver suture before resection, F, G - surgical resection of renal tumour, $\mathbf{H}, \mathbf{I}$ - renal parenchyma suturation after tumour resection, $\mathbf{J}$ - locating the drain, $\mathbf{K}$ - removing the tumour, $\mathrm{L}$ - image of the resected tumour 
tumours were present in 1 female patient. The tumour was located on the right side in $70.5 \%(12 / 17)$ of the patients. The R.E.N.A.L nephrometry score showed the location of the tumour in the kidney in all patients. The mean R.E.N.A.L. score was 5.3, and the mean tumour diameter was measured as 2.72 $(1.6-4.4) \mathrm{cm}$. No need to return to open operation or Clavien-Dindo class 3-4 complications were observed in any of the patients. The mean operation duration was 126 (81-180) min, the hospitalisation period was $49.6(28-114) \mathrm{h}$ and the bleeding amount was 244 (50-400) ml. Clavien-Dindo class 1-2 complications were observed in 4 (25\%) patients. Gastric dilatation recovering with nasogastric catheter application was observed in 1 patient; a Clavien-Dindo class 1 complication caused by wound area infection recovering with antibiotic treatment was observed again in 1 patient; and Clavien-Dindo class 2 complications caused by blood transfusion were observed in 2 patients. Patients were followed up for 16.2 months on average. No findings suggestive of local

Table I. Patient-related, peroperative, postoperative and histopathological findings $(n=16)$ (average (distribution) or number unless the opposite is stated)

\begin{tabular}{|lc|}
\hline Parameter & Result \\
\hline Age & $61.7(49-72)$ \\
\hline Tumour diameter [cm] & $2.72(1.6-4.4)$ \\
\hline Gender & $\begin{array}{c}11 \text { males/5 females } \\
(68.75 \% / 31.25 \%)\end{array}$ \\
\hline Right/left ratio & $12 / 5(70.5 \% / 29.5 \%)$ \\
\hline R.E.N.A.L. score & $5.3(4-7)$ \\
\hline Need to return to open operation & 0 \\
\hline Operation duration [min] & $126(81-180)$ \\
\hline Hospitalisation [h] & $49.6(28-114)$ \\
\hline Bleeding amount [ml] & $244(50-400)$ \\
\hline Clavien-Dindo class 1-2 complication & $4(25 \%)$ \\
\hline Clavien-Dindo class 3-4 complication & 0 \\
\hline Follow-up period [months] & 16.2 \\
\hline Clear cell carcinoma & $11(64.7 \%)$ \\
\hline Chromophobe cell carcinoma & $3(17.6 \%)$ \\
\hline Papillary cell carcinoma & $2(11.7 \%)$ \\
\hline Cystic cell carcinoma & $1(5.8 \%)$ \\
\hline
\end{tabular}

recurrence or metastasis were detected in any of the patients.

The surgical border was reported to be negative in all extracted tumours. The distance of the tumour from the surgical border was measured as $2 \mathrm{~mm}$ in 1 patient. Pathology was reported as clear cell carcinoma in 11 (64.7\%) patients, papillary cell carcinoma in 2 (11.7\%) patients, chromophobe cell carcino$\mathrm{ma}$ in $3(17.6 \%)$ patients and cystic cell carcinoma in $1(5.8 \%)$ patient. It was observed that all tumours were low grade. Patient-related, peroperative, postoperative and histopathological findings can be seen in Table I.

The preoperative mean creatinine and eGFR levels were found to be $0.77 \pm 0.17 \mathrm{mg} / \mathrm{dl}$ and 99.7 $\pm 14.2 \mathrm{ml} / \mathrm{min}$, respectively. The 1 -day postoperative mean creatinine and eGFR levels were measured as $0.88 \pm 0.14 \mathrm{mg} / \mathrm{dl}$ and $92.3 \pm 10.6 \mathrm{ml} / \mathrm{min}$. The 12-month postoperative mean creatinine and eGFR levels were measured as $0.83 \pm 0.08 \mathrm{mg} / \mathrm{dl}$ and 95.7 $\pm 10.9 \mathrm{ml} / \mathrm{min}$. Although the changes in postoperative creatinine and eGFR values were clinically insignificant, the difference between pre- and postoperative values was statistically significant $(p=0.033$, $p=0.045$, respectively). While the difference between the preoperative and 1-day postoperative creatinine and eGFR values was detected to be statistically significant when the mutual analysis of data was carried out ( $p=0.039, p=0.042$, respectively), a statistically significant difference was not detected among preoperative and 12-month postoperative creatinine and eGFR values ( $p=0.09, p=0.065$, respectively). Global percent functional recovery was measured as $92.5 \%$ on day 1 and as $95.9 \%$ after 12 months. Statistical values and data for creatinine and eGFR values can be seen in Table II.

\section{Discussion}

The fact that the most important factor determining the condition of kidney functions after partial nephrectomy is preoperative GFR level has been reported in many studies [4, 10]. Generally, when evaluating the patients, the value called global percent functional recovery is used to calculate how postoperative GFR has been preserved compared to preoperative GFR, expressed as a percentage. Although this value changes according to the surgical method and ischaemia application after PN, it has been reported as $76-86 \%$ in many studies [4]. It has been demonstrated that radical nephrectomy (RN) 
Table II. Statistical values and data for creatinine and eGFR values

\begin{tabular}{|lcccccc|}
\hline Parameter & Preoperative & $\begin{array}{c}\text { Postoperative } \\
\text { day } 1\end{array}$ & $\begin{array}{c}\text { Postoperative } \\
\text { month } 12\end{array}$ & $P$-value* & $\begin{array}{c}\text { Preop.-postop. } \\
\text { day 1 } \\
P \text {-value* }\end{array}$ & $\begin{array}{c}\text { Preop.- postop. } \\
\text { month } 12 \\
P \text {-value* }\end{array}$ \\
\hline $\begin{array}{l}\text { Creatinine } \\
{[\mathrm{mg} / \mathrm{dl}]}\end{array}$ & $0.77 \pm 0.17$ & $0.88 \pm 0.14$ & $0.83 \pm 0.08$ & 0.033 & 0.039 & 0.09 \\
\hline $\begin{array}{l}\text { eGFR } \\
{[\mathrm{ml} / \mathrm{min}]}\end{array}$ & $99.7 \pm 14.2$ & $92.3 \pm 10.6$ & $95.7 \pm 10.9$ & 0.045 & 0.042 & 0.065 \\
\hline
\end{tabular}

${ }^{*}$ Friedman's two-way analysis of variance.

increases chronic kidney disease and cardiovascular disease risk and negatively affects patients' general survival. Thus, the need for MIPN, especially in T1 phase tumours, has gained importance [11]. The most important factor affecting renal function loss after PN is warm ischaemia time (WIT). Currently, MIPN is generally accomplished through the clamping of hilar veins.

Many authors have described their experiences with off-clamp, clampless or unclamped MIPN in the past decade. Although these procedures create a risk for increased blood loss, they minimise ischaemic renal damage and the loss of renal functions. While first experiences were gained through controlled hypotension and superselective arterial microdissection, many modified methods have been defined over time [12].

Thompson et al. identified a WIT threshold value considering postoperative acute kidney insufficiency (AKI) development risk as 25 min. Every minute, an increase over this value demonstrates a 5-6\% increase in AKI [13]. Moreover, Rais-Bahrami et al. compared functional results of patients who underwent MIPN with the odd-clamp (126 patients) and hilar-clamp (264 patients) techniques; they reported a changing ratio in percentages of $\mathrm{s} C \mathrm{r}$ levels in favour of the off-clamp procedure [14]. Thus, we avoided warm ischaemia to preserve the kidney functions of our patients. We believe that warm ischaemia will have a negative effect on kidney functions in the long term.

In the Papalia et al. study, although complication rates were determined to be significantly higher in the $>4 \mathrm{~cm}$ group compared to the group with a tumour dimension $\leq 4 \mathrm{~cm}$ or the group with tumours $>4 \mathrm{~cm}$ in which laparoscopic and robotic MIPN was applied without renal ischaemia in 121 patients, no significant difference could be detected in postoperative renal scintigraphy controls [15]. In our patients, we especially considered a tumour dimension $\leq 4 \mathrm{~cm}$, as this was appropriate for our technique and in or- der to decrease the complication rates. We believe that that R.E.N.A.L nephrometry 9-point system had a successful design when it came to considering whether our patients were suited for this treatment.

Gill et al. described their laparoscopic and robotic MIPN approach involving clamping hilar veins; moreover, they coined the term 'zero ischaemia' in the literature [16]. Zero ischaemia can be accomplished with different procedures, such as selective/segmental renal artery clamping, selective renal parenchymal clamping, cutting off the targeted renal blood flow, laser-supported MIPN, radiofrequency-supported MIPN, hydrojet-supported MIPN and the sequential preplaced suture renorrhaphy technique [17].

Forbes et al. reported that there was an $\mathrm{s} C \mathrm{r}$ increase of 9.2 in the early postoperative period in $21 \mathrm{pa}$ tients who underwent zero-ischaemia robotic-assisted PN. They stated that this situation was related to the dimension of the mass extracted and intraoperative renal ischaemia [18]. However, no data were provided on long-term kidney results in this study. In another study, it was found that the main effect on preserving late-period renal functions was due to the preservation of a high renal volume [19].

Hung et al. examined patients undergoing PN with discovery-era hilar-occlusion WIT $(n=139)$, conventional hilar-occlusion WIT $(n=213)$, limitedischaemia WIT $(n=104)$ and zero-ischaemia $(n=78)$ techniques in their study of 534 patients. Although the parenchymal mass percentage in all patients was preserved, with a ratio of $88-90 \%$, global percent functional recoveries were measured as $80 \%$, $79 \%, 89 \%$ and $91 \%$, respectively, using these techniques. In this study, the highest renal function protection percentage was measured in the zero-ischaemia group [20].

$\mathrm{Ng}$ et al. found that median global function was preserved with a ratio of $86 \%$ in 44 patients who underwent assisted and laparoscopic zero-ischaemia PN [21]. This ratio was detected as $95.9 \%$ in 
our study, and the difference was related to the fact that vascular microdissection was carried out in this group, and patients with bigger tumours and higher R.E.N.A.L. nephrometry scores were chosen.

There are two studies in which PN was reported with the sequential preplaced suture renorrhaphy technique. This technique was initially described by Rizkala et al., who applied it in 14 patients with a mean tumour dimension of $2.2 \mathrm{~cm}$; in this study, the mean bleeding amount was $192.5 \mathrm{ml}$ and the mean operation time was 160 min. Clavien-Dindo grade 3-4 complications were not reported in any of the patients, and surgical border positivity was not determined in the patients. When compared to the preoperative results, an $8.1 \%$ decrease in eGFR ratio was detected in the early postoperative period in this study, while long-term results were not mentioned [22]. In our study, the decrease in postoperative eGFR levels was measured as $7.5 \%$ on day 1 and $4.1 \%$ at 12 months after the operation; these results were in line with the previous study.

The second study was carried out by Lu et al., and zero ischaemia was employed in 13 out of 14 patients. Here, the mean bleeding amount was 60 (30-200) $\mathrm{ml}$, and the average operation duration was 75 min. No major complications occurred in any of the patients, and neither local recurrence nor metastasis was observed [23]. Although postoperative kidney functions were stated to be normal in this study, no data on value interval and long-term kidney functions were reported.

In our study, the mean tumour dimension was $2.72 \mathrm{~cm}$, the mean bleeding amount was $244 \mathrm{ml}$, and the mean operation time was $126 \mathrm{~min}$. No Clavien-Dindo grade 3-4 complications occurred in any of the patients, and surgical border positivity was not determined. These results are in line with the 2 previous studies described above. Although the changes in postoperative creatinine and eGFR values were clinically insignificant, the differences between pre- and postoperative values were statistically significant $(p=0.033, p=0.045$, respectively). This result revealed that renal function could be significantly affected even with nephron-protecting surgery without causing ischaemia. Therefore, we suggest that use of zero-ischaemia MIPN is very important for the preservation of long-term kidney functions in suitable patients.

Our study showed that the modified sequential preplaced suture renorrhaphy technique is a suc- cessful method for preserving long-term kidney functions. It is useful and applicable in patients suited for PN. Moreover, it has a low complication rate and good oncological results.

Our study had several limitations. We only considered tumours under $4 \mathrm{~cm}$ and the R.E.N.A.L. scores were low. Moreover, we focused on an exophytic tumour type with no relationship with the collecting system and that was not close to the renal hilus. Although the technique used is rather successful in preserving long-term kidney functions, we do not believe that it is applicable in kidney tumours of all types and dimensions. We believe that undesired results, such as complications and surgical border positivity, may be seen in patients with large tumour dimensions and high R.E.N.A.L. scores.

We did not perform perioperative ultrasonography during surgery or frozen section biopsy from the base of the tumour, as suggested in the literature, because these procedures could prolong the operation time, increase the amount of bleeding and morbidity and have negative effects on long-term kidney functions. As kidney tumours are generally encapsulated, we suggest that these interventions are not required in a PN procedure performed by preserving capsule integrity and leaving a safe surgical border. A good preoperative radiological evaluation, crossing the needle from the optimal localisation during surgery and rapid suture ligation after the tumour is completely resected are important aspects when this surgical technique is applied, and it is necessary for the team to be experienced in these aspects. As none of our patients had surgical border positivity after the operation, this surgical technique seems to be successful for complete resection of the tumour. We also believe that comparative studies including more patients and a longer follow-up period are needed.

\section{Conclusions}

The modified sequential preplaced suture renorrhaphy technique is an effective, reliable method to prevent complications, improve oncological results and preserve renal functions and nephrons in appropriate, selected patients. This method avoids the damage caused by warm ischaemia on the kidney. However, due to its limitations, it can only be applied in certain patients with kidney tumours. As the number of patients in whom the technique is applied in- 
crease, we believe that more reliable results may be achieved.

\section{Conflict of interest}

The authors declare no conflict of interest.

\section{References}

1. Ljunberg B, Bensalah K, Bex A, et al. Guidelines on Renal Cell Carcinoma. European Association of Urology Guidelines 2015 edition.

2. Kural AR, Demirkesen O, Önal B, et al. Outcome of nephron-sparing surgery: elective versus imperative indications. Urol Int 2003; 71: 190-6.

3. Luciani LG, Cestar R, Tallarigo C. Incidental renal cell carcinoma - age and stage characterization and clinical implications: study of 1092 patients. Urology 2000; 56: 58-62.

4. Mir MC, Ercole C, Takagi T, et al. Decline in renal function after partial nephrectomy: etiology and prevention. J Urol 2015; 193: 1889-98.

5. Winfield HN, Donovan JF, Godet AS, Clayman RV. Laparoscopic partial nephrectomy: initial case report for benign disease. J Endourol 1993; 7: 521-6.

6. Gill IS, Delworth MG, Munch LC. Laparoscopic retroperitoneal partial nephrectomy. J Urol 1994; 152: 15339-42.

7. Pettus JA, Sharp DS, Yossepowitch O, et al. Tumor location does not affect long-term renal function after partial nephrectomy. Urology 2007; 69: 1059-63.

8. Arslan B, Önol şY, Değirmenci T, et al. Comparison of pedicle clamping and parenchymal clamping in patients with renal cell carcinoma during partial nephrectomy. Ege J Med 2013; 52: 147-51.

9. Kutikov A, Uzzo RG. The R.E.N.A.L. nephrometry score: a comprehensive standardized system for quantitating renal tumor size, location and depth. J Urol 2009; 182: 844-53.

10. Mir MC, Takagi T, Campbell RA, et al. Poorly functioning kidneys recover from ischemia after partial nephrectomy as well as strongly functioning kidneys. J Urol 2014; 192: 665-70.

11. Huang WC, Elkin EB, Levey AS, et al. Partial nephrectomy versus radical nephrectomy in patients with small renal tumors - is there a difference in mortality and cardiovascular outcomes? J Urol 2009; 181: 55-62.

12. Simone G, Ferriero M, Papalia R, et al. Zero-ischemia minimally invasive partial nephrectomy. Curr Urol Rep 2013; 14: 465-70.

13. Thompson RH, Lane BR, Lohse CM, et al. Every minute counts when the renal hilum is clamped during partial nephrectomy. Eur Urol 2010; 58: 340-5.

14. Rais-Bahrami S, George AK, Herati AS, et al. Off-clamp versus complete hilar control laparoscopic partial nephrectomy: comparison by clinical stage. BJU Int 2011; 109: 1376-81.

15. Papalia R, Simone G, Ferriero M, et al. Laparoscopic and robotic partial nephrectomy without renal ischaemia for tumours larg. er than $4 \mathrm{~cm}$ : perioperative and functional outcomes. World J Urol 2012; 30: 671-6.

16. Gill I, Eisenberg MS, Aron M, et al. "Zero Ischemia" partial nephrectomy: novel laparoscopic and robotic technique. Eur Urol 2011; 59: 128-34
17. Hou W, Ji Z. Achieving zero ischemia in minimally invasive partial nephrectomy surgery. Int I Surg 2015; 18: 48-54.

18. Forbes E, Cheung D, Kinnaird A, Martin BS. Zero ischemia robotic-assisted partial nephrectomy in Alberta: initial results of a novel approach. Can Urol Assoc J 2015; 9: 128-32.

19. Simmons MN, Fergany AF, Campbell SC. Effect of parenchymal volume preservation on kidney function after partial nephrectomy. J Urol 2011; 186: 405-10.

20. Hung AJ, Cai J, Simmons MN, Gill IS. "Trifecta" in partial nephrectomy. J Urol 2013; 189: 36-42.

21. Ng CK, Gill IS, Patil MB, et al. Anatomic renal artery branch microdissection to facilitate zero ischemia partial nephrectomy. Eur Urol 2012; 61: 67-74.

22. Rizkala ER, Khalifeh A, Autorino R, et al. Zero ischemia robotic partial nephrectomy: sequential preplaced suture renorrhaphy technique. Urology 2013; 82: 100-4.

23. Lu J, Zu Q, Du Q, et al. Zero ischaemia laparoscopic nephronsparing surgery by re-suturing. Contemp Oncol 2014; 18: 355-58.

Received: 1.01.2017, accepted: 23.03.2017. 